\title{
Article
}

\section{A Static Hybrid Renewable Energy System for Off-Grid supply}

\author{
Augusto Montisci ${ }^{1}$, Marco Caredda ${ }^{2}$ \\ 1,* University of Cagliari, Italy augusto.montisci@unica.it \\ 2 Independent Researcher; marco.caredda@gmail.com
}

\begin{abstract}
The electrification of rural areas of the planet has become one of the greatest challenges for sustainability. In fact, it would be the key to guaranteeing development for the poorest of the planet, but from which most of the raw material for the food market derives. The paradigm of centralized production is not applicable in these territories, because the distribution network would involve unjustifiable costs. For this reason, studies have multiplied to ensure the energy supply, especially electricity, of off-grid utilities, to guarantee energy autonomy while reducing the dependence on specialist assistance for the management of the system. In this work, a hybrid system (HRES) is proposed that combines the exploitation of solar energy with that of the wind through the use of static devices, in order to improve the system's availability and limit the cost of operation and maintenance.
\end{abstract}

Keywords: HRES; Energy static conversion; Off-grid supply; Thermoacoustics

\section{Introduction}

The problem of sustainability has recently taken on prominence on the agenda of almost all national governments and international institutions, both because of the increasingly evident human impact on the ecosystem with increasingly dramatic consequences, and behind the push of public opinion world increasingly aware of the risks involved. Unfortunately the policies adopted so far have limited themselves to allocating financial resources to programs of a still very general nature, but there is still a long way to go to significantly affect the footprint. In fact, what has been achieved so far has been only to reduce the relationship between footprint and economic growth, but the challenge that humanity must face today is to reverse the trend, while guaranteeing the resources for all the populations that inhabit the whole planet. A simple reduction in consumption would be feasible, but not likely. In fact, the states that consume the most are also those with the greatest political weight, and continue to adopt policies that place sustainability in the background to economic growth. While waiting for the states to take note of the fact that the economy cannot grow indefinitely, a change in the growth paradigm itself can be made immediately, shifting the focus on quality rather than quantity. This is possible because awareness of the inadequacy of economic indicators is growing more and more, and that real well-being is often in counter-trend with respect to economic wealth. Energy is probably the sector in which it is easier to raise awareness among the population and at the same time the one in which results can be obtained more easily. In fact, nowadays there is a very wide gap between the ways in which energy is used and the available technology. Reducing this gap would result in an immediate reduction in the consumption of resources and in the impact that their use entails, but for a period of time that can be estimated in a few decades, this would produce economic growth rather than decrease. It has been amply demonstrated that the replacement of fossil fuels with renewables involves an increase in the number of people employed, and even if the turnover of companies is reduced, the economic balance benefits from it. Furthermore, the growth in employment feeds the market, which does not lead to a worsening of the footprint, as long as the entire economy is sustainable. The use of renewables is recognized as one of the fundamental factors for implementing a sustainable economy, but it is not the only one. 
The real keyword is "smartness" or the reasonableness in identifying technical solutions. For example, today the centralized energy model is increasingly in crisis, in which a power plant produces energy and then distributes it extensively to a network of users on a national and often supranational scale. The limitations of this model are mainly three: the primary source is normally a fossil fuel which is not extracted near the power plant, therefore it is necessary to spend energy for transport; the power plant requires the exclusive use of a much wider area than the plant, having to ensure respect distances; third, the transmission and distribution network is an extremely expensive infrastructure, and it is estimated that $20 \%$ of the total electricity produced is lost in distribution. It is therefore understandable why there is more and more interest in solutions such as smart grids, where the three problems described, rather than being solved, are eliminated at the root.

The study presented in this work is part of this trend, but with particular attention to the specific problem of the electrification of off-grid areas [1,3,6,8-11,15,16,18-20,22-28, 30,32-36,39,40,42,44,46-48]. Electricity is considered a fundamental driver of development, but in many rural areas of the planet the creation of a distribution network would be completely unjustified. The literature proposes numerous studies concerning the supply of off-grid utilities or microgrids using renewable sources. Not surprisingly, these studies focus mostly on rural areas of Africa and India, and on areas with a large number of islands, such as the Philippines [3,6,15,19, 21-25, 27,28,30-32,34,36,39,44,45]. The problems that must be faced are the low density and discontinuity of the primary source and the cost of energy storage systems $[1,4,5,7,17]$. There are many tools that allow one to cope with these difficulties. The main one is to make consumption smart, that is, to make use of efficient devices, to consume energy for real needs, to adapt the timing of consumption to limit the need to store energy.

Having said this, the energy system must maximize its sustainability, both in environmental and economic terms $[2,6,11,21,23,43-45]$. The sun is by far the most widely used primary source, both to produce heat and electricity. In domestic installations and small smart grids, photovoltaics is used almost exclusively [1, 5,16,17,20 22,30,31,36,38,43], while thermodynamic solar is commonly used in power plants. The choice of smart solutions requires that thermal users are powered by converting solar radiation directly into heat, rather than powering an electric heater powered by electricity. At the same time it would be reasonable to carry out cooling by directly exploiting the heat, but this type of solution is less common. Another primary source widely used also in residential areas is wind [1,43,56-58]. The market offers various solutions for vertical axis micro-wind turbines, installed on the ground. Other solutions, such as geothermal energy, sea energy, rivers, are applicable only in special cases, but on a global scale they can offer an important contribution. Perhaps the most problematic aspect is represented by energy storage. The options are many (batteries, ultracapacitors, flywheels, counterweights, heat tanks, ...), but each has limitations, so optimal load management is extremely important. To mitigate the storage problem, several hybrid solutions have been proposed in the literature, in which different primary sources and different storage systems are incorporated into a single system (HRES [1,10-14,17,20,24,25,27,31,37,40,42-48]). The most immediate advantage derives from the fact that the different sources are not correlated with each other, therefore in a multi-source system the intervals during which there is no production are shorter, and therefore the necessary capacity can be reduced.

In this work an HRES system is proposed with the peculiar characteristic of using only static devices both for the conversion of the primary source and for storage and recovery. The rationale for this choice is, in general, to increase the overall efficiency of the system, because frictions are eliminated and higher temperatures can be managed, but also because the robustness of the system increases, and therefore the availability and lifecycle, which for an isolated building are of fundamental importance. The rest of the article is organized as follows. Section 2 describes the processes and devices used for both conversion and storage. In section 3 the layout as a whole is presented, and a maximum sizing of the system is indicated. Some final considerations conclude the article. 


\section{Materials and Methods}

The guiding idea of this study is to use static devices for the conversion of energy deriving from different types of primary sources, as well as for their storage and recovery. Static energy conversion processes have always attracted the attention of both the research world and industry, due to the obvious advantages: absence of friction and wear, longer life cycle duration, reduced maintenance, possibility to operate in a contaminated environment and to use sources at extremely high temperatures, higher power density, both with respect to volume and mass, greater operational flexibility. Nonetheless, only a limited number of these processes have been adopted on a large scale, each of them for different problems, but all pay for the fact that there is already an alternative technology that has had a strong development. For this reason, the comparison between traditional and innovative static mobile technologies must be made in perspective, based on the potential for future evolution that can be hypothesized for each. Photovoltaics deserves a separate discussion. It is in fact a static technology that converts solar radiation into electricity in a single stage, so it would seem to have all the requirements to be included in the system we are proposing here. The fact is that static is the only requirement that this technology possesses among those required here, as it lacks in our opinion in terms of sustainability. In fact, the cost per kWh is very high, the yield decays in a few years, the maintenance is expensive, it is not suitable for operating in different environments, it presents decommissioning problems, and finally the balance between energy produced in the life cycle and that expense to build a panel is roughly even. For this reason, it was preferred in this work to analyse alternative technologies for the conversion of solar energy, which, as will be seen below, have further important advantages.

This section will analyse in detail the components that will then be combined with each other to obtain the energy autonomous building described above. Some of the technologies described below have already reached a very advanced level of development for some time, also by their commercial success has made substantial investments possible. Other components are still at a low TRL, but have nevertheless been included in this work as they possess, compared to traditional competitors, better characteristics of sustainability, both from a technical and an economic point of view. This is the case, for example, of the thermoacoustic generator, preferred to photovoltaics for the exploitation of solar energy by virtue of the lower cost, or the use of resonant cavities instead of turbines for the exploitation of wind energy. This choice, although it may seem like a gamble in the case of an investment, offers interesting prospects, and for this reason it is believed to be worth analysing. The prospect is to obtain a highly integrated and completely static hybrid system, which overall should guarantee the system a considerably higher technical-economic sustainability standard than that of the HRES systems proposed in the literature.

\subsection{Solar concentrators}

Solar energy is used here for three different types of utilities, namely heat generation, refrigeration, and electricity generation. Regardless of the utility to be powered by solar radiation, it is always advisable to concentrate it in advance $[49,50]$. Ideally this solution could also be used for photovoltaics, because for the same amount of captured energy the active surface would be reduced. Nonetheless, in practice it is preferred not to adopt this type of solution, because photovoltaic cells pose serious cooling problems, which in this way would be accentuated, even if there is no lack of studies and experiments in this direction [51], often combining the production of electricity with heat. This problem does not exist when the entire spectrum of solar radiation contributes to the transformation process, as in the cases examined in this work. Through non-imaging concentrators it is possible to concentrate the solar radiation in order to reach high temperatures, with benefit of the efficiency. Different types of concentrators exists [50], while in this work the Fresnel lenses and mirrors are used, which compared to parabolic and hyperbolic concentrators have the advantage of being smaller. The main difference between mirrors and 
lenses is that in the latter the radiation must pass through a medium, which can never be completely transparent. On the other hand, with mirrors, the focal point is visible, resulting in a visual impact and possible maintenance problems. The receiver, positioned in the focus of the concentrator, must be able to withstand very high temperatures without rapidly degrading, and at the same time must limit the energy radiated due to the temperature. If the temperature of the receiver remains low, one can think of exploiting the greenhouse effect, encapsulating the receiver inside a vacuum tube in a material that is transparent to solar radiation but opaque with respect to the radiation emitted by the receiver itself. As the temperature rises, the overlap between the solar spectrum and the emission spectrum of the receiver becomes greater and greater, so a compromise must be found between the energy lost by radiation of the receiver and the solar energy that does not reach the receiver because it is filtered from glass. In this case it is preferred to insert the receiver inside a cavity, so that the ratio between emitted energy and incident energy depends on the geometry of the cavity. The in-depth study of this element goes beyond the scope of this study, so we will limit ourselves to assuming that the system can reach a temperature of $500^{\circ} \mathrm{C}$, referring to the specific literature for the design aspects.

\subsection{Heat loads}

The water temperature for domestic utilities is much lower than that which can be reached even with a simple solar thermal panel. Conversely, in the case of electricity or refrigeration production, there is an interest in maximizing the temperature at the receiver. For this reason, it makes sense to combine the different utilities powered by the sun, so that the resulting heat from the two high-temperature processes feeds the users that require hot water (domestic water, kitchen, washing machine, dishwasher, etc. $\left(40^{\circ} \mathrm{C}-100^{\circ} \mathrm{C}\right)$ ). To make the system simple, it is assumed that a single heat accumulation system at a constant temperature $\left(110^{\circ} \mathrm{C}\right)$ is prepared, made with molten salts, with the precaution of avoiding substances that are hazardous to human health.

\subsection{Generazione elettrica alimentata con energia solare}

As an alternative to photovoltaics, by virtue of lower costs and greater efficiency, in this work the thermoacoustic process (TA) has been preferred. The thermo-acoustic effect [5155] was discovered by the Phoenician glass blowers not later than the first century BC, but only recently the phenomenon has been interpreted. Experimentally, they observed that, when the difference of temperature at the ends of the duct overcame a critical value, a sound was produced. The phenomenon is quite simple: the hot wall transfers some heat to a particle of gas, which as a consequence enlarges its volume, touches a colder part of the wall and then it returns the heat to the wall, reducing its volume and assuming the initial position. The oscillation of the particles is perceived by our ear as a sound, but the most important aspect is that it represents a conversion of heat into mechanical energy (gas vibration), so it performs as an engine, but without solid moving parts. In order for the phenomenon to last over time, it is necessary to ensure that the two ends of the duct are kept at a constant temperature, so it will be necessary to ensure an efficient heat exchange both on the high temperature end and on the other low temperature one. The low temperature heat exchange can take place with air, or with a fluid of exchange. Since the efficiency of the thermoacoustic conversion depends on the temperature gradient and not on the absolute value, an element, called regenerator, is usually inserted inside the resonant tube, made of a material that conducts little heat, and with a shape that ensures the gas particles to swing freely in the longitudinal direction with respect to the tube. The adoption of the regenerator allows to trigger the thermoacoustic effect even with modest temperature jumps $\left(100^{\circ} \mathrm{C}\right)$, it being understood that the conversion efficiency will be the higher the greater the overall temperature jump. Another fundamental aspect concerns the gas that is used as an operating fluid. Since the gas is contained within the resonator, 
it is possible to choose it so that its properties are better suited to the process. Typically, the gases used for this type of device are helium and argon.

After the first thermoacoustics stage, in order to obtain electrical current, a further transformation is necessary, which can be again performed statically, by means of piezoelectric crystals, or by transferring the TA vibration to a solid component. The former solution has the advantage that the entire conversion process is static, but the level of power is limited. On the other hand, interfacing the TA stage with an alternate cycle, such as a Stirling engine, the level of power is higher, but the static nature of the whole apparatus is compromised. An easy solution for this stage consists of using a loudspeaker in reverse mode, in the sense that the vibration moving the magnet of the cone induces an electrical current in the coil.

\subsection{Solar cooling}

The thermoacoustic effect is reversible, in the sense that by means of a sound vibration it is possible to pump the heat, as in a cooling cycle. This means that, coupling a thermoacoustic resonator with a thermoacoustic cooler, the energy of the sun can be used for cooling. This solution is much more preferable to that of producing electrical energy and then to supply a conventional appliance, because many energy conversion steps are avoided, with the benefit of the efficiency and the cost, and furthermore the simplicity of the thermoacoustic device is exploited to perform the entire transformation from the source (sun) to the utility.

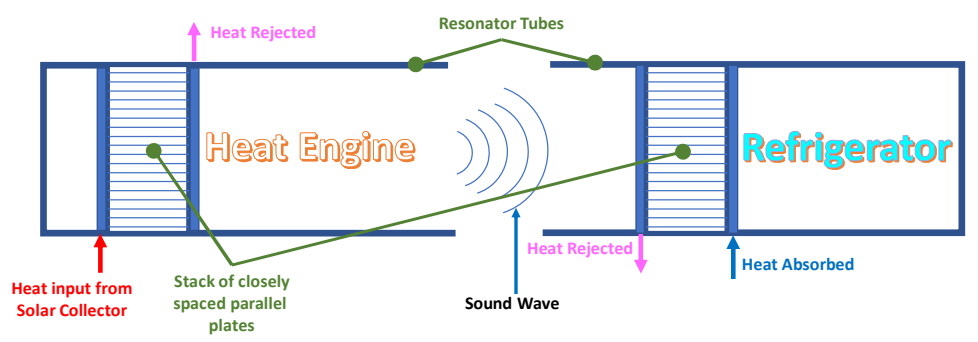

Figure 1. Thermoacoustics-baed cooling system

As previously mentioned, an energy storage system is mandatory, in order to guarantee the continuity of the service during the night. Nonetheless, an emergency option has to be envisaged, in case the energy reserves should be terminated or compromised. The thermoacoustics has the advantage that it works with heat, no matter how this heat has been produced. This means that in case of emergency, the solar radiation could be temporarily substituted with contingency reserves of fuel or, for example, wood.

\subsection{Wind power}

The possibility of tapping into different primary sources of energy is of fundamental importance for an off-grid system. In fact, any renewable source remains unavailable for shorter or longer periods, during which it is necessary to make up for it through reserves previously accumulated or through conventional generation systems, typically the generator set. In both cases, the periods of unavailability of the primary source generally 
represent the greatest obstacle to overcome for the realization of a totally autonomous system.

Typically, the wind energy is preferred in extra urban areas, because of the better ratio between produced energy and used land with respect to other renewable energy sources. Therefore, the integration of wind energy conversion systems into buildings is a small but growing trend, and it has a great scope in generating electricity from the wind. In fact, in Europe there is increasing interest from home owners and businesses to install small wind turbines on the rooftop of houses, buildings of school, hospitals, commercial buildings, or even on tall buildings [56]. Small wind turbines are particularly suitable for distributed production of electrical energy. Combining solar and wind systems allows one to mitigate the problem of the discontinuity of the primary source, which is typical for both the sources alone. On the other hand, it is important to improve the ratio between the encumbrance and the power of the system. In [57], a system is proposed which exploits the building to convey the wind towards a small turbine placed in the under roof. This innovative system allows one to avoid the typical drawbacks of wind generation, such as the encumbrance of the blades, noise, cut-in and cut-off velocities of the wind, turbulence, etc. Such layout entails several advantages. Firstly, the visual impact and safety problems of the turbine are definitively avoided, because it is hidden inside the structure. Secondly, the wind direction is not a problem, because the vertical axis of the rotor allows it to capture the wind no matter which direction it comes from. Thirdly, the stator allows one to handle a wide section of flow, possibly the entire building can be used to intercept the wind, even if the dimension of the turbine is very small, depending on the ratio between the inlet and the outlet cross section of the stator. This allows one to exploit a wider range of wind velocities with respect to common wind generators, because of a lower cut in and unlimited cut off velocities.

The power associated with the wind flow is given by the formula:

$P=\frac{1}{2} \rho A v^{3}$

where $P$ represents the power, $\rho$ is the air density, $A$ is the cross section of the flow tube, $v$ is the wind speed. From (1) it emerges that the time diagram of wind speed significantly affects the power associated with the wind. More specifically, it can be seen that with the same average speed, the power available is greater the more the speed values are variable. For this reason, the site where a wind power plant is installed is preferably characterized with the Weibull diagram [58], which provides the probability density function of the wind speed as a function of two parameters that characterize the site. It follows that the possibility of processing the wind, whatever its speed, determines a significant variation of the electrical power produced, especially in case the statistical distribution of the speeds has a significant tail beyond the cut-off speed. The system proposed in [57], due to its small size and the fact that it is constrained into a cage, allow the turbine to rotate at much higher regimes of any ordinary wind turbine. Of the kinetic power of the wind, only a part can ideally be converted by a wind turbine, because the flow out of the turbine cannot be stopped, otherwise the conversion would cease. From Betz's law we know that the 
maximum power is obtained when the output speed is equal to $1 / 3$ of the input speed. In the case described in [57] the Betz theory is not immediately applicable, because a centripetal turbine is adopted, in which the conversion of the wind power is not linked to the reduction of the speed, but thanks to the change of direction from horizontal to vertical. For this type of system it is not possible to formulate a theory of general validity, as the properties of the incoming and outgoing flow depend on the boundary conditions, and therefore in the case in question on the shape of the building and the orography of the land. Nonetheless, the different way of processing the flow compared to conventional wind turbines favors the conversion process, so it can be assumed that the limit set by Betz's law can be exceeded.

In this work, in order to create an entirely static system, the turbine is replaced by resonant cavities [59]. In this way wind energy is converted into acoustic power, exactly as it happens for solar energy, so it is possible to integrate the two sources to create a single transformation system, both for electricity and for refrigeration. The evaluation of the conversion efficiency in this case is even more complex, due to the high number of degrees of freedom in the sizing of the resonant cavities. The sizing of the system goes beyond the aims of this study, which only aims to provide an order of magnitude of the system parameters.

\subsection{Energy storage}

The energy storage system is necessary because the plant is off-grid. As mentioned above, the need to store energy is mitigated by the fact that two different primary sources are used, which reduces the time intervals in which no external source is available. The need for a smaller reserve is reflected in a lower cost of the storage system. The static and hybrid nature of the system is also adopted for energy conservation. The variety of storage systems allows to size each device in order to contain both costs and space. For this reason, in principle it would be convenient to use non-static devices, such as flywheels and counterweights, but for consistency with the static nature of the system, these solutions are not taken into consideration in this work. Batteries are also excluded for different reasons, due to their limited lifespan and the disposal problems they entail. For these reasons, the storage systems that will be considered will be the combination of a cycle based on hydrogen, ultracapacitors, biomass (mainly wood), an accumulation system based on a fluid with high thermal capacity. The individual modules are briefly described individually below.

\subsubsection{Hydrogen energy storage}

Although the hydrogen-based storage system has a high cost compared to all the other components of the plant, it has the advantage of no other limitation on the amount of energy than that of the hydrogen containers. Excess electricity powers an electrolyser that decomposes water into oxygen and hydrogen molecules. To draw from the energy reserve, the hydrogen is oxidized by means of a fuel cell, obtaining a direct current, which can eventually be transformed into power by the inverter. For both the electrolyser and the fuel cell, the market offers a wide range of solutions of different size, efficiency and therefore price. It is important that in the dimensioning of the hydrogen storage system the real need is not exceeded, also taking into account the other storage systems, in order to avoid an unnecessary increase in costs. The hydrogen system also has the advantage of not predicting the release of greenhouse gases into the atmosphere. A lower cost solution, 
but also less efficient, is the one that provides for the combustion of hydrogen, feeding the same thermo-acoustic system that converts solar energy. This solution allows to significantly reduce investment costs because the fuel cell is replaced with a simple burner, but while the former can reach an efficiency of up to $70 \%$, the efficiency of the thermo-acoustic cycle is around $10 \%$. This implies that in order to guarantee a sufficient reserve of energy, it will be necessary to increase the power of the plant, as well as provide for a greater volume of hydrogen storage. Furthermore, it is not recommended to use air as an oxidizer for hydrogen, because nitrogen oxides are created, which are among the main causes of acid rain. Since ideally the stoichiometric amount of oxygen necessary for the combustion of hydrogen is obtained as a by-product of hydrolysis, it is sufficient to store this oxygen and use it for combustion. However, this requires a greater volume for oxygen storage, and the burner is also more complex because the combustion temperature is significantly higher.

\subsubsection{Ultracapacitors}

Ultracapacitors (UC) [60] represent an alternative to batteries, as they store electrical energy through the electric field, rather than through a chemical reaction. Although the power density of these devices is not competitive with respect to batteries, they have very significant advantages, such as the possibility of carrying out charge / discharge cycles in very short times, without any consequence on the duration of the life cycle, which is significantly longer than the batteries. The market offers numerous alternatives of ultracapacitors, which can be installed and used in the same way as a battery. In combination with the hydrogen system mentioned in the previous paragraph, the UCs allow better management of current transients both in the hydrogen production phase and in the use of reserves. If used for this purpose, the required capacity becomes modest, and the cost too. Alternatively, it is possible to think about the operating principle of the UCs, to build a system capable of storing much greater quantities of energy. The energy stored in a capacitor is given by the formula:

$$
E=\frac{1}{2} C V^{2}
$$

where $E$ is the energy, $C$ the capacity and $V$ the potential difference between the armatures of the capacitor. The technology of UCs, compared to that of traditional capacitors, has made it possible to increase the energy that can be stored by some orders of magnitude. This result was obtained thanks to some modifications in the realization, which allowed to increase the capacity $C$. This is expressed by the relation:

$$
C=\varepsilon \frac{A}{d}
$$

where $C$ is the capacity, $\varepsilon$ is the permittivity of the insulating material placed between the reinforcements, $A$ is the area of the two reinforcements, $d$ the distance that separates them. The increase in capacity was obtained by intervening on the two parameters of the area and the distance. To increase the former, the capacitor plates are made of porous material, so that the active surface can be up to 3000 times the value of the external surface. On the other hand, to reduce the distance $d$, an electrolyte with a suitable concentration of ions is inserted in place of the dielectric between the plates. In this way, when the voltage $V$ is applied between the plates, the ions of the electrolyte are attracted to the armature with opposite polarity, thus obtaining a double layer capacitor, i.e. two capacitors in series where the two intermediate plates are constituted from the electrolyte ions. To allow the ions to get as close as possible to the two plates, they are not electrically isolated, so that the distance $d$ separating the plates is almost reduced to an atomic scale. Precisely to avoid the short circuit between the armature and ions, the operating voltage 
of the capacitor must be small, typically $6 \mathrm{~V}$. To obtain higher voltage values, the capacitors are connected in series.

In this work, it was hypothesized to exploit the operating principle of the UCs to create a system that is not currently available on the market, but which apparently does not present particular implementation difficulties. The idea is to use ultra-capacitive panels to cover all the internal surfaces of the house, such as the walls, the ceiling, the floor. While using a less sophisticated technology than that with which the UCs currently on the market are made, and therefore limiting costs, it is possible to create a storage system with a capacity far superior to that of any normal battery system. Keeping in mind the principle of sustainability, which pushes not to oversize the plants with respect to the needs, and by virtue of the fact that the use of different primary sources reduces the need to store energy, it can be hypothesized that such a system can alone provide the storage capacity needed by a domestic user. Nonetheless, to increase the resilience of the entire system, it is considered appropriate to provide for the adoption of different storage technologies

\subsubsection{Biomass}

Especially in poorer countries, biomass, mainly firewood, is still the most used primary source of energy. In various regions of the world this has a negative impact on the environment, especially in areas where a process of desirtification is underway. For this reason, attempts are made to favor the transition to other forms of energy, sometimes even preferring fossil fuels. Taking this aspect into account, biomass has not been included among the main sources of the proposed system, but we are limited to considering it as a reserve source. We distinguish here the wet biomass from the dry one. The use of the former does not cause the impact on the environment mentioned above, and indeed its use for energy purposes mitigates the problem of waste disposal. The simplest way to use wet biomass for energy purposes is to use an anaerobic digester, which produces a biogas that can be burned to produce the heat with which to feed the thermoacoustic cycle, or to power thermal utilities (sanitary water, kitchen, wash). In the same way, dry biomass can also be used. Given the modest amount that is required, it is possible to predict that this biomass may derive from pruning, and therefore does not create the negative effects mentioned above. In this work, to avoid overly complicating the layout of the plant, it was assumed to use only dry biomass, therefore without the need to set up an anaerobic digester.

\section{Layout of the overall system}

This section presents a working hypothesis of a static HRES system for meeting the energy needs of an isolated housing unit. While respecting the constraints set out above, regarding the exclusive use of static devices, and the attention to sustainability both from a technical-economic and environmental point of view, the definition of the system has wide margins of discretion. Furthermore, the design solution strictly depends on the climate of the area in which the project is carried out, on the specific energy demand of the user, from which materials and which professional skills are most easily accessible. Having said this, it is clear that any layout presented here can only represent an initial draft, which would require several changes to adapt it to any real context. 


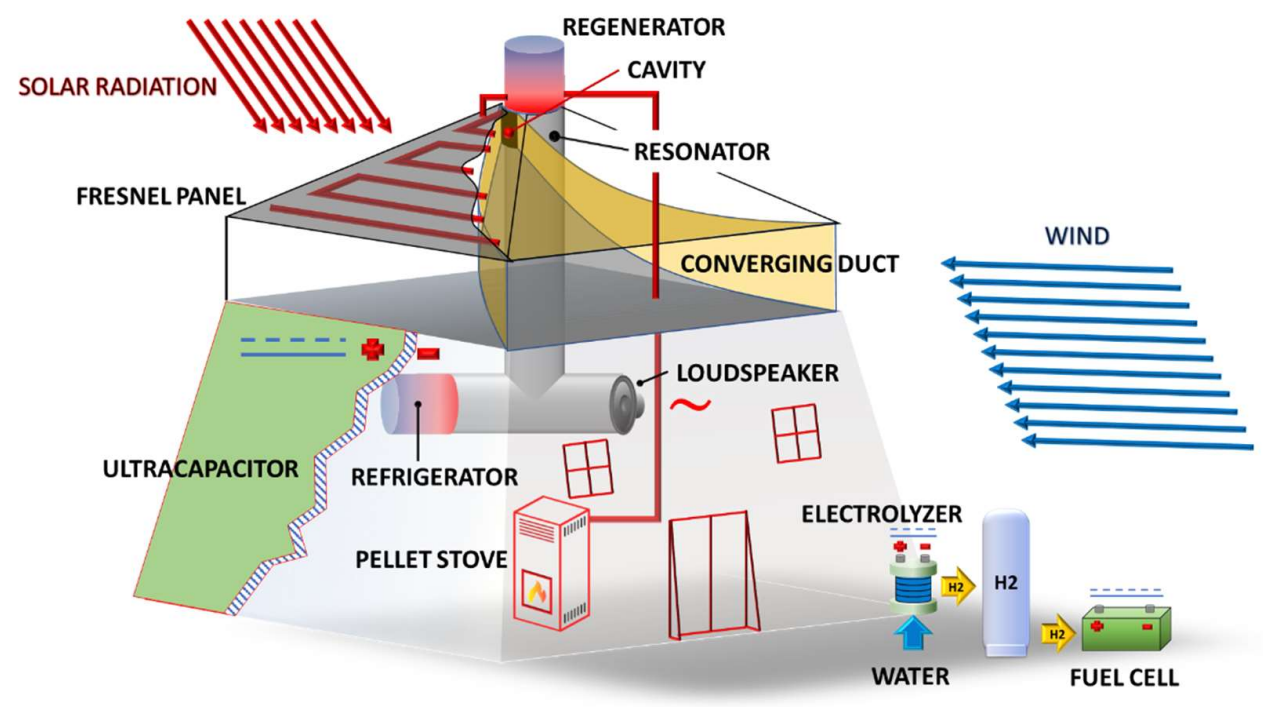

Figure 2: Functional scheme of the proposed HRES system

Fig. 2 shows a functional diagram of the integrated system. The roof cover is made up of Fresnel lenses that heat a coil through which the thermoacoustic generator is powered. The heat taken from the cold end has a sufficient temperature to power the building's thermal utilities (sanitary water, heating, washing). It is possible to switch the thermoacoustic resonator to choose whether to produce electricity through the loudspeaker or to power a second thermoacoustic resonator for direct production of cold (food preservation, conditioning). The thermo-acoustic system can also be powered by a pellet stove, which works as a backup on the main reserve represented by hydrogen. The volume below the roof is divided into converging ducts to convey the wind towards the resonant cavity in the central column. The walls of the building are shaped in such a way to converge the flow of wind towards the roof and at the same time limit turbulence at the inlet to the duct. In the diagram in Fig. 2 for simplicity, only one of the four ducts is reported. The wind resonant cavity is interfaced with the thermoacoustic resonator, in order to share the next stage of conversion of the acoustic power.

When the electricity produced exceeds consumption, it can be stored both by means of the ultra-capacitor panels and by powering an electrolyzer for the production of hydrogen. Ultracapacitors are suitable for storing small amounts of energy and in the presence of strong transients, both in the charging and discharging phases. Conversely, hydrogen allows to store a much greater amount of energy, but requires more regular operation. The hydrogen produced by the electrolyser is stored in a special container under pressure and used to power the fuel cell when consumption exceeds production.

\subsection{System Dimensioning}

The sizing of the system is particularly complex, unless to oversize it. First of all, it is necessary to consider the hourly diagram of consumption in the different periods of the year, also taking into account the hourly flexibility of the loads, which allow to limit the amount of energy to be stored. Preliminarily it is assumed that the energy demand is equal to $36 \mathrm{MJ} /$ day, divided between electrical, thermal and refrigeration consumptions, without further specification on the hourly diagram, even if it is reasonably assumed that consumption is more concentrated during daylight hours. 
Subsequently it is necessary to evaluate the producibility of energy from sun and wind. Solar energy has the advantage of regularity, which favors sizing, but has the disadvantage of being available for a limited period within the 24 hours. Depending on the geographical area, solar radiation can be more or less relevant. In this study, it was assumed to build the plant on the island of Sardinia, in the Mediterranean, where the average value of daily solar radiation is about $25 \mathrm{MJ} / \mathrm{m}^{2}$. It is assumed that the Fresnel panels completely cover the roof of the building, and that during the hours of sunshine the surface exposed to solar radiation is equal to $10 \mathrm{~m}^{2}$, for a total of $250 \mathrm{MJ} / \mathrm{day}$. On the basis of literature data, an efficiency of $90 \%$ of the concentration, $30 \%$ of the thermoacoustic conversion and finally $37 \%$ of the loudspeaker is assumed, with an overall efficiency equal to $10 \%$, for which $25 \mathrm{MJ}$ /day are obtained to power the electrical utilities. Part of this energy is absorbed for refrigeration, for which an overall efficiency similar to that of electricity generation is assumed as a first approximation. $90 \%$ of the thermal energy processed by the thermoacoustic generator is recovered on the cold side of the regenerator at a temperature of around $100{ }^{\circ} \mathrm{C}$, therefore suitable for powering thermal users. By assuming a lower temperature on the cold side of the regenerator, it is possible to slightly increase the efficiency of the thermoacoustic stage, therefore by modulating the supply temperature of the thermal utilities it is possible to vary the amount of electricity produced.

To correctly size the wind system, as mentioned above, it is necessary to consider numerous elements, such as the fluid dynamic characteristics of the building, the orography of the surrounding area, the wind regime. As a first approximation, it is assumed that the building is able to convey a flow tube of $20 \mathrm{~m}^{2}$ into the converging duct. The overall wind energy depends on the distribution of speeds, while the wind direction does not affect the process. On the basis of in situ monitoring data it was possible to define the following parameters of the Weibull distribution of the wind at zero altitude: $k=1.7$, $\lambda=4$.2. Assuming the Betz formula, a mean wind energy value of $114 \mathrm{MJ} /$ day is obtained. In this work, cautiously one assumes that the conversion efficiency of kinetic energy into vibration is $50 \%$ and considering that the loudspeaker is the same used in the thermoacoustic system (37\% efficiency), an additional electrical energy value of $21 \mathrm{MJ} /$ day is obtained by the wind system. This means that on average the electricity generated would theoretically be sufficient to power all the loads. This is not the reality, as the surplus of energy over the average consumption is necessary to cope with the losses in storage.

The smart organization of loads takes on fundamental importance, because as long as it is possible, it is better not to use storage. The loads must be divided between those that can be deferred and that ones which cannot be, and ensure that the latter are active in the hours in which there is greater production of electricity. This means that the loads must be scheduled mainly during the day, limiting the night loads as much as possible. At the same time, it is useful to have a certain number of loads that can be activated at any time the wind is blowing, therefore possibly even at night.

The part of production that exceeds the consumption is directed to the storage system. This is done in three different ways, namely the ultra capacitor panels, the integrated electrolyser+fuel cell system, pellet stove. None of the three systems has the characteristics to be adopted as the only solution, but their complementarity allows them to be combined effectively.

The ultra-capacitive panels have very low losses in the charge/discharge cycle and can withstand very high currents, which makes them suitable for managing current peaks, both in the charging and discharging phases. The commercial ultracapacitors can reach energy densities up to $200 \mathrm{MJ} / \mathrm{dm}^{3}$, but this value is not plausible for capacitive panels 
such as those foreseen in this study. Considering non-engineered systems made in the laboratory, an energy density of $1 \mathrm{MJ} / \mathrm{dm}^{3}$ can be considered plausible. Assuming to realize a paneling of dimensions $100 \times 20 \times 0.1 \mathrm{dm}^{3}$, one obtains a system capable of storing about $200 \mathrm{MJ}$, which for the working hypotheses correspond to about 5.5 days of autonomy. This value cannot be considered sufficient, as the maximum consumption occurs precisely in the winter period, when the availability of solar energy is reduced, while the energy of the wind is not very different from that which occurs in the summer, therefore the exhaustion of this reserve is a plausible hypothesis. Theoretically, the number of panels could be increased, but the solution appears excessively expensive. More accurate economic data will be possible when panels such as those described in this paper will be available on the market.

The part of the energy to be stored that exceeds the capacity of the panels is accumulated in the form of Hydrogen by means of an electrolyser. For the Electrolyser+Fuel Cell system there are various solutions on the market, even for domestic solutions. In this work it was assumed to use a $2.2 \mathrm{~kW}$ electrolyser, capable of producing up to $0.5 \mathrm{~m} 3 /$ hour of hydrogen, which correspond to $6.22 \mathrm{MJ}$, which in turn can be converted into $3.24 \mathrm{MJ}$ by means of the fuel cell. The amount of energy that can be stored depends on the hydrogen storage volume. This represents the long-term energy reserve, and serves to compensate for the imbalance between production and consumption, which typically occurs during the winter, therefore the sizing of the storage volume must be calibrated considering the possibility of having to accumulate energy for the 'winter. It should also be considered that the overall efficiency of the complete charge/discharge cycle is about $41 \%$, while in the case of panels the efficiency is close to unity, therefore the use of this form of storage must be evaluated carefully and only to the extent request.

The third mode for energy storage involves a pellet stove, which as biomass is a primary source of energy, but is not produced within the system. With this system it is possible to power the thermoacoustic resonator in place of the sun, and in this way the electric utilities are powered. The system is considered in this work for emergencies, in the event that the other two reserves are exhausted, but it does not fall within the energy balance of the system, which is sized in such a way that the two aforementioned systems are sufficient to guarantee to meet the demand of energy.

\section{Conclusions}

In this work, an HRES system for the energy supply of an off-grid building is presented. The sources used are solar radiation and wind, both of which are converted into acoustic power respectively through a thermoacoustic resonator and a resonant cavity. The sound energy can in turn be transformed into electrical energy through a loudspeaker, or for cooling through a second thermoacoustic resonator coupled in reversible mode with the first. The excess energy produced is stored through a dual system consisting of ultracapacitive panels, which are used for short-term storage, and a combined electrolyser + fuel cell system, which guarantee the reserve in the inter-seasonal period. Finally, there is an emergency reserve consisting of a pellet stove with which it is possible to power the thermoacoustic resonator and then power the entire system. The study is still in a preliminary phase and requires experimental verification, but offers promising prospects for the electrification of isolated users.

Conflicts of Interest: The authors declare no conflict of interest.

\section{References}

1. A. M. Patel and S. K. Singal, "Off grid rural electrification using integrated renewable energy system," 2016 IEEE 7th Power India International Conference (PIICON), 2016, pp. 1-5, doi: 10.1109/POWERI.2016.8077272. 
2. P. E. Campana, L. Wästhage, W. Nookuea, Y. Tan, J. Yan, Optimization and assessment of floating and floating-tracking PV systems integrated in on- and off-grid hybrid energy systems, Solar Energy, 2019, vol. 177, pp. 782-795

3. K. Trapani, D. L. Millar, Proposing offshore photovoltaic (PV) technology to the energy mix of the Maltese islands, Energy Conversion and Management, 2013, vol. 67, pp. 18-26

4. M. Redón Santafé, J. B. Torregrosa Soler, F. J. Sánchez Romero, P. S. Ferrer Gisbert, J. J. Ferrán Gozálvez, C. M. Ferrer Gisbert, Theoretical and experimental analysis of a floating photovoltaic cover for water irrigation reservoirs, 2014, Energy, vol. 67, pp. 246-255,

5. R. Cazzaniga, M. Cicu, M. Rosa-Clot, P. Rosa-Clot, G.M. Tina, C. Ventura, Compressed air energy storage integrated with floating photovoltaic plant, Journal of Energy Storage, 2017, vol. 13, pp. 48-57

6. P. P Kumar, R. P. Saini, Optimization of an off-grid integrated hybrid renewable energy system with different battery technologies for rural electrification in India, Journal of Energy Storage, 2020, vol. 32, 101912

7. M. Jafari, D. Armaghan, S.M. S. Mahmoudi, A. Chitsaz, Thermoeconomic analysis of a standalone solar hydrogen system with hybrid energy storage, International Journal of Hydrogen Energy, 2019, vol. 44, Issue 36, pp. 19614-19627

8. J. M. Aberilla, A. Gallego-Schmid, L. Stamford, A. Azapagic, Design and environmental sustainability assessment of small-scale off-grid energy systems for remote rural communities, Applied Energy, 2020, vol. 258, 114004

9. A. de Almeida, P. Moura, and N. Quaresma, Energy-efficient off-grid systems-review. 2020, Energy Efficiency, vol. 13, pp. 349376

10. Y.S. Mohammed, M.W. Mustafa, N. Bashir, Hybrid renewable energy systems for off-grid electric power: Review of substantial issues, Renewable and Sustainable Energy Reviews, 2014, vol. 35, pp. 527-539

11. R. Siddaiah, R.P. Saini, A review on planning, configurations, modeling and optimization techniques of hybrid renewable energy systems for off grid applications, Renewable and Sustainable Energy Reviews, 2016, vol. 58, pp 376-396

12. K. S. Krishna, K. S. Kumar, A review on hybrid renewable energy systems, 2015, Renewable and Sustainable Energy Reviews, vol. 52, pp. 907-916

13. A. Mahesh, K. S. Sandhu, Hybrid wind/photovoltaic energy system developments: Critical review and findings, Renewable and Sustainable Energy Reviews, 2015, vol. 52, pp. 1135-1147

14. S. Goel, R. Sharma, Performance evaluation of stand alone, grid connected and hybrid renewable energy systems for rural application: A comparative review, Renewable and Sustainable Energy Reviews, 2017, vol. 78, pp. 1378-1389

15. C. L. Azimoh, P. Klintenberg, F. Wallin, B. Karlsson, C. Mbohwa, Electricity for development: Mini-grid solution for rural electrification in South Africa, Energy Conversion and Management, 2016, vol. 110, pp 268-277

16. D. O. Akinyele, R. K. Rayudu, Comprehensive techno-economic and environmental impact study of a localised photovoltaic power system (PPS) for off-grid communities, Energy Conversion and Management, 2016, vol. 124, pp. 266-279

17. A. Singh, P. Baredar, B. Gupta, Techno-economic feasibility analysis of hydrogen fuel cell and solar photovoltaic hybrid renewable energy system for academic research building, Energy Conversion and Management, 2017, vol. 145, pp 398-414

18. S. C. Bhattacharyya, S. Ohiare, The Chinese electricity access model for rural electrification: Approach, experience and lessons for others, Energy Policy, 2012, vol. 49, pp. 676-687

19. H. Meschede, E. A. Esparcia, P. Holzapfel, P. Bertheau, R. C. Ang, A. C. Blanco, J. D. Ocon, On the transferability of smart energy systems on off-grid islands using cluster analysis - A case study for the Philippine archipelago, Applied Energy, 2019, vol. 251, 113290

20. S. Bensmail, D. Rekioua, H. Azzi, Study of hybrid photovoltaic/fuel cell system for stand-alone applications, International Journal of Hydrogen Energy, 2015, vol. 40, Issue 39, pp. 13820-13826

21. C. Mokhtara, B. Negrou, N. Settou, A. Bouferrouk, Y. Yao, Design optimization of grid-connected PV-Hydrogen for energy prosumers considering sector-coupling paradigm: Case study of a university building in Algeria, International Journal of Hydrogen Energy, 2020, in press

22. P. Sandwell, N. L. A. Chan, S. Foster, D. Nagpal, C. J.M. Emmott, C. Candelise, S. J. Buckle, N. Ekins-Daukes, A. Gambhir, J. Nelson, Off-grid solar photovoltaic systems for rural electrification and emissions mitigation in India, Solar Energy Materials and Solar Cells, 2016, vol. 156, pp. 147-156

23. P. Bertheau, P. Blechinger, Resilient solar energy island supply to support SDG7 on the Philippines: Techno-economic optimized electrification strategy for small islands, Utilities Policy, 2018, vol. 54, pp. 55-77

24. H. El-houari, A. Allouhi, S. Rehman, M.S. Buker, T. Kousksou, A. Jamil, B. El Amrani, Feasibility evaluation of a hybrid renewable power generation system for sustainable electricity supply in a Moroccan remote site, Journal of Cleaner Production, 2020, vol. 277, 123534

25. A. C. Brent, D. E. Rogers, Renewable rural electrification: Sustainability assessment of mini-hybrid off-grid technological systems in the African context, Renewable Energy, 2010, vol. 35, Issue 1, pp. 257-265

26. A.B. Kanase-Patil, R.P. Saini, M.P. Sharma, Integrated renewable energy systems for off grid rural electrification of remote area, Renewable Energy, 2010, vol. 35, Issue 6, pp. 1342-1349

27. J.G. Castellanos, M. Walker, D. Poggio, M. Pourkashanian, W. Nimmo, Modelling an off-grid integrated renewable energy system for rural electrification in India using photovoltaics and anaerobic digestion, Renewable Energy, 2015, vol. 74, pp. 390-398

28. N. Ramchandran, R. Pai, A. K. S. Parihar, Feasibility assessment of Anchor-Business-Community model for off-grid rural electrification in India, Renewable Energy, 2016, vol. 97, pp. 197-209 
29. K. Ulsrud, T. Winther, D. Palit, H. Rohracher, J. Sandgren, The Solar Transitions research on solar mini-grids in India: Learning from local cases of innovative socio-technical systems, Energy for Sustainable Development, 2011, vol. 15, Issue 3, pp 293-303

30. M. Millinger, T. Mårlind, E.O. Ahlgren, Evaluation of Indian rural solar electrification: A case study in Chhattisgarh, Energy for Sustainable Development, 2012, vol. 16, Issue 4, pp. 486-492

31. M. S. Adaramola, S. S. Paul, O. M. Oyewola, Assessment of decentralized hybrid PV solar-diesel power system for applications in Northern part of Nigeria, Energy for Sustainable Development, 2014, vol. 19, pp. 72-82

32. J. Urpelainen, Grid and off-grid electrification: An integrated model with applications to India, 2014, Energy for Sustainable Development, vol. 19 , pp. 66-71

33. S. Martin, J. Susanto, Supplying power to remote villages in Lao PDR. - The role of off-grid decentralised energy options, Energy for Sustainable Development, 2014, vol. 19, pp. 111-121

34. G. Veilleux, T. Potisat, D. Pezim, C. Ribback, J. Ling, A. Krysztofiński, A. Ahmed, J. Papenheim, A. M. Pineda, S,. Sembian, S. Chucherd, Techno-economic analysis of microgrid projects for rural electrification: A systematic approach to the redesign of Koh Jik off-grid case study, Energy for Sustainable Development, 2020, vol. 54, pp. 1-13

35. B. Mainali, S. Silveira, Using a sustainability index to assess energy technologies for rural electrification, Renewable and Sustainable Energy Reviews, 2015, vol. 41, pp. 1351-1365

36. S. Baurzhan, G. P. Jenkins, Off-grid solar PV: Is it an affordable or appropriate solution for rural electrification in Sub-Saharan African countries?, Renewable and Sustainable Energy Reviews, 2016, vol. 60, pp. 1405-1418

37. H. Rezk, G. M. Dousoky, Technical and economic analysis of different configurations of stand-alone hybrid renewable power systems - A case study, Renewable and Sustainable Energy Reviews, 2016, vol. 62, pp. 941-953

38. C. Ghenai, T. Salameh, A. Merabet, Technico-economic analysis of off grid solar PV/Fuel cell energy system for residential community in desert region, International Journal of Hydrogen Energy, 2020, vol. 45, Issue 20, pp. 11460-11470

39. L. Lozano, E. M. Querikiol, M. L. S. Abundo, L. M. Bellotindos, Techno-economic analysis of a cost-effective power generation system for off-grid island communities: A case study of Gilutongan Island, Cordova, Cebu, Philippines, Renewable Energy, 2019, vol. 140, pp. 905-911

40. SK.A. Shezan, S. Julai, M.A. Kibria, K.R. Ullah, R. Saidur, W.T. Chong, R.K. Akikur, Performance analysis of an off-grid windPV (photovoltaic)-diesel-battery hybrid energy system feasible for remote areas, Journal of Cleaner Production, 2016, vol 125, pp. 121-132

41. P. Halder, E. Paladinić, M. Stevanov, S. Orlović, T. J. Hokkanen, P. Pelkonen, Energy wood production from private forests nonindustrial private forest owners' perceptions and attitudes in Croatia and Serbia, Renewable and Sustainable Energy Reviews, 2014, vol. 35, pp. 515-526

42. T. Tezer, R. Yaman, G. Yaman, Evaluation of approaches used for optimization of stand-alone hybrid renewable energy systems, Renewable and Sustainable Energy Reviews, 2017, vol. 73, pp. 840-853

43. K. Anoune, M. Bouya, A. Astito, A. B. Abdellah, Sizing methods and optimization techniques for PV-wind based hybrid renewable energy system: A review, Renewable and Sustainable Energy Reviews, 2018, vol. 93, pp. 652-673

44. C. Mokhtara, B. Negrou, A. Bouferrouk, Y. Yao, N. Settou, M. Ramadan, Integrated supply-demand energy management for optimal design of off-grid hybrid renewable energy systems for residential electrification in arid climates, Energy Conversion and Management, 2020, vol. 221, 113192

45. S. Mandal, B. K. Das, N. Hoque, Optimum sizing of a stand-alone hybrid energy system for rural electrification in Bangladesh, Journal of Cleaner Production, 2018, vol. 200, pp. 12-27

46. P. Rullo, L. Braccia, P. Luppi, D. Zumoffen, D. Feroldi, Integration of sizing and energy management based on economic predictive control for standalone hybrid renewable energy systems, Renewable Energy, 2019, vol. 140, pp. $436-451$

47. R. Hosseinalizadeh, H. Shakouri G, M. S. Amalnick, P. Taghipour, Economic sizing of a hybrid (PV-WT-FC) renewable energy system (HRES) for stand-alone usages by an optimization-simulation model: Case study of Iran, Renewable and Sustainable Energy Reviews, 2016, vol. 54, pp. 139-150

48. V. Suresh, M. Muralidhar, R. Kiranmayi, Modelling and optimization of an off-grid hybrid renewable energy system for electrification in a rural areas, Energy Reports, 2020, vol 6, pp. 594-604So

49. C.F. Chen, C.H. Lin, H.T. Jan, Y.L. Yang, Design of a solar concentrator combining paraboloidal and hyperbolic mirrors using ray tracing method, Optics Communications, 2009, vol. 282, Issue 3, pp. 360-366,

50. R. Winston, J.C. Minano and P. Benitez, Nonimaging Optics, Elsevier Academic Press, 2005, pp. 1-217.

51. K. Wang, D. Sun, J. Zhang, Y. Xu, J. Zou, K. Wu, L. Qiu, Z. Huang, Operating characteristics and performance improvements of a 500W traveling-wave thermoacoustic electric generator, Applied Energy, 2015, vol. 160, pp. 853-862

52. O. G. Symko and I. A. Rodríguez, Acoustic approach to thermal management, 2010, 12th IEEE Intersociety Conference on Thermal and Thermomechanical Phenomena in Electronic Systems, pp. 1-4,

53. H. Kang, P. Cheng, Z. Yu, H. Zheng, A two-stage traveling-wave thermoacoustic electric generator with loudspeakers as alternators, Applied Energy, 2015, vol. 137, pp. 9-17

54. ASTER website, available online http://www.aster-thermoacoustics.com/ (accessed on 30 July 2021)

55. G.W. Swift, Thermoacoustics, Springer, 2017

56. J. Cace, et al., Urban wind turbines: Guidelines for small wind turbines in the built environment, Wineur Intelligent Energy Europe, 2007, pp. 1-41 
57. S. Carcangiu and A. Montisci, A building-integrated eolic system for the exploitation of wind energy in urban areas, 2012, ENERGYCON 2012

58. S. E. Tuller, and A. C. Brett. The Characteristics of Wind Velocity That Favor the Fitting of a Weibull Distribution in Wind Speed Analysis, 1984, Journal of Climate and Applied Meteorology, vol. 23, no. 1, pp. 124-134

59. A. Rona, The acoustic resonance of rectangular and cylindrical cavities, Journal of Algorithms E Computational Technology, 2007, Vol. 1, no. 3, pp. 329-355

60. Petar J. Grbovic, Ultra-Capacitors in Power Conversion Systems, John Wiley \& Sons, 2013 